DOI: $10.3901 / J M E .2020 .13 .041$

\title{
煤机设备无线自供电状态监测系统 ${ }^{*}$
}

\author{
高 扬 ${ }^{1}$ 穆继亮 ${ }^{1}$ 何 剑 $^{1}$ 陈 鸿 $^{1}$ 穆锦标 ${ }^{1}$ \\ 安站东 $^{2}$ 赵新平 $^{2}$ 丑修建 $^{1}$
}

(1. 中北大学仪器科学与动态测试教育部重点实验室 太原 030051;

2. 阳煤集团机电动力部 阳泉 045000)

\begin{abstract}
摘要: 针对当下煤机设备状态监测系统时效性差、通信电缆铺设困难、需要定期更换电池等问题, 提出了一种基于无线传感 器网络与自供电技术的监测系统设计方法。系统由能量采集模块、无线传感器网络及监测上位机组成, 通过能量采集模块收 集煤机设备振动能量为节点电路供电, 采用无线传感器网络与上位机实现设备关键状态信息的采集、传输与处理。测试结果 表明, 系统可在 $30 \mathrm{~m}$ 有效范围内实现对煤机设备运行状态的实时监测, 能量采集模块最大输出功率可达 $378 \mathrm{~mW}$, 能够有效 收集设备振动能量实现系统能源自供给。
\end{abstract}

关键词: 煤机设备; 自供电技术; 无线传感器网络; 监测系统

中图分类号: TD76; TP274

\section{Wireless Self-powered Condition Monitoring System for Coal Machine Equipment}

\author{
GAO Yang ${ }^{1}$ MU Jiliang $^{1}$ HE Jian $^{1}$ CHEN Hong ${ }^{1} \quad$ MU Jinbiao $^{1}$ AN Zhandong ${ }^{2}$ \\ ZHAO Xinping ${ }^{2}$ CHOU Xiujian ${ }^{1}$
}

(1. Key laboratory of Instrumentation Science \& Dynamic Measurement of Ministry of Education, North University of China, Taiyuan 030051;

2. Department of Electromechanical Power, Yangquan Coal Group, Yangquan 045000)

\begin{abstract}
The Wireless sensor network and self-powered technology-based monitoring system was proposed to solve the several problems, such as the poor timeliness of coal machinery equipment condition monitoring system, the difficulty in laying communication cables, and the demand to replace batteries regularly. The system consists of energy harvesting unit, wireless sensor network unit and monitoring software unit; the energy harvesting unit can harvest the mechanical energy of vibration to supply power for the node circuit; the wireless sensor network unit and monitoring software unit realize the collection, transmission and processing of critical state information of equipment. The system can realize the real-time monitoring of machine equipment operating status within $30 \mathrm{~m}$ effective range and the maximum output power of energy harvesting unit can reach up to $378 \mathrm{~mW}$, which can effectively collect equipment vibration energy and realize system energy self-supply.
\end{abstract}

Key words: coal machine equipment; self-powered technology; wireless sensor network; monitoring system

\section{0 前言}

煤炭是我国的主要能源, 在国民经济中占有重 要战略地位, 煤矿机械化水平直接影响着煤矿的生 产效率和经济效益 ${ }^{[1-2]}$ 。近些年来, 因操作不规范、 设备陈旧老化、检修不到位等因素带来的煤矿机械

* 国家自然科学基金资助项目(51675493)。20190818 收到初稿, 20191019 收到修改稿
事故频发, 保证煤机设备安全可靠运行显得尤为重 要。建立煤机设备在线状态监测系统获取设备关键 状态信息进行预知维修, 是提高煤矿生产效率和保 障安全生产的有效途径 ${ }^{[3-4]}$ 。

受恶劣工作环境影响, 煤机设备不可避免会产 生各种机械故障。故障往往表现为异常的振动和温 度变化, 这些振动与温度信号中包含了大量设备由 于冲击、磨损、疲劳、老化等因素引起的劣化和失 效等重要设备状态信息, 是设备状态在线监测与故 障诊断的重要数据来源 ${ }^{[5-6]}$ 。对煤机设备工作过程中 
振动和温度信号进行实时监测, 可提早发现设备故 障, 实现由 “事后维修” 到 “预知维修” 的转变, 从而有效避免突发性事故发生, 消除续发损坏, 节 约大量维护费用 ${ }^{[7-8]}$ 。

煤机设备具有旋转、强振动等特点, 传统的线 缆供电、数据传输的监测方式存在铺设成本高与线 缆缠绕等问题而无法广泛使用 ${ }^{[9]}$ 。恶劣的工作环境 也容易导致线缆老化和损坏, 无线传感器网络 (Wireless sensor network, WSN)通过集成微电子技 术、嵌入式计算、现代网络及无线通信和分布式信 息处理等技术，可以有效地解决上述问题。一般 WSN 中传感器节点需由电池供电, 定期更换电池成 本高且在实际应用中具有很强的局限性。采用自供 电技术收集环境能量为传感器节点供电的方式是解 决这一难题的有效途径 ${ }^{[10]}$ 。从 20 世纪末开始, 机 械、电气、材料等领域的很多研究人员展开了对微 能源器件的探索研究。近年来, 将环境能量转换为 电能的发电模式得到了各国科学家的青睐。WANG 等 ${ }^{[11-13]}$ 对微能源的基础理论、结构和发展形式进行 了深入探索与研究。在人体健康、生态环境和工业 设备监测等领域中，自供电技术展现出了良好的研 究和应用前景 ${ }^{[14-15]}$ 。

本文针对煤机设备状态监测系统发展现状与技 术瓶颈, 提出集能量自供给、多节点数据采集、信 号无线传输、上位机实时监测等多功能为一体的煤 机设备无线自供电在线监测系统研究。对提高煤机 监测效率、提升煤炭工业生产效率、降低煤矿机械 安全事故率、提升煤矿重型机械装备的智能化改造 具有一定的应用价值和现实意义。

\section{1 系统总体方案}

监测系统由信号采集节点、数据接收器、系统 上位机组成。其中信号采集节点是整个系统的最前 端, 内置自供电模块、电路模块、无线传输模块等 功能单元。如图 1 所示, 在煤机设备的关键部位安 装信号采集节点, 通过能量采集模块与能量管理电 路, 转化并收集设备运行时的振动能量为节点电路 供电。利用传感器电路对设备的温度、振动信息进 行实时采集、处理。通过无线传输模块将传感数据 发送至数据接收器, 之后采用串口通信的方式将数 据传输至上位机软件, 经数据解码、节点号识别与 信号分析, 对传感数据进行存储、处理和分析。最 终在控制面板中实现设备运行状态的实时监测和预 警，完成监测系统的人机交互。

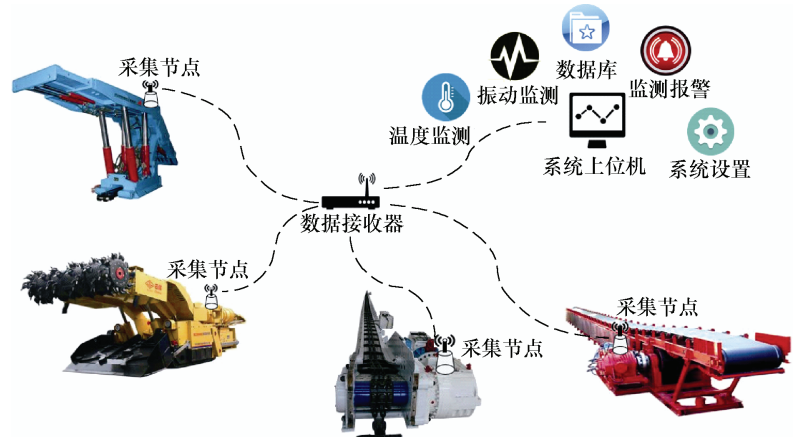

图 1 系统应用示意图

\section{2 系统硬件设计}

监测系统硬件主要包括能量采集模块、能源管 理电路、主控电路、传感器模块以及无线传输模块。 如图 2 所示, 通过能量采集模块将煤机设备振动能 量转化为电能, 之后利用能量管理电路对转换得到 的电能进行有效收集和存储, 继而为节点电路供电; 传感器模块进行煤机设备关键位置振动与温度信息 的实时采集; 主控芯片控制各个模块运行并对传感 器采集到的信号进行处理; 无线传输模块用以实现 数据的远程通信。

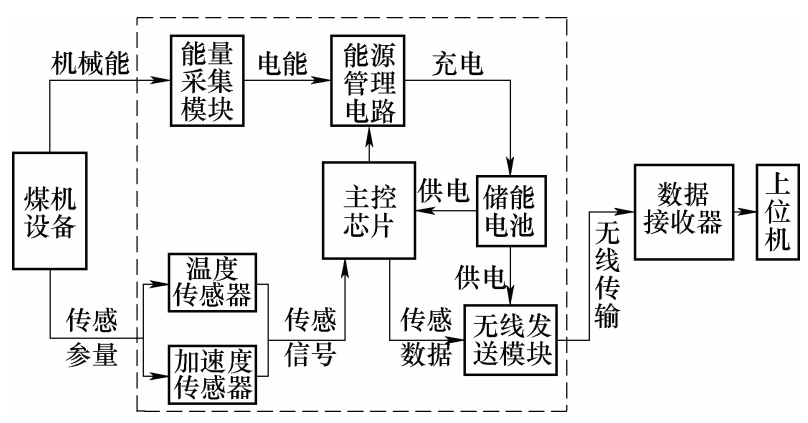

图 2 系统工作流程图

\section{1 能量采集模块设计}

自供电技术是将系统所处周围环境中风能、热 能、太阳能和机械能等各种能源通过能量收集装置 转化为电能, 为电路系统供电的一种技术 ${ }^{[16-17]}$ 。收 集环境能量以解决信号采集节点的供电问题具有广 阔的研究前景和应用价值 ${ }^{[18]}$ 。考虑到能量来源和转 换效率, 热能、风能以及太阳能等能源很难在矿井 之中进行有效收集。而当煤机设备运行时，机身表 面会产生振动, 该振动不仅能够反应机器的工作状 态，亦可作为一种稳定的能量来源。采用自供电技 术收集机器振动的能量为采集节点电路供电具有很 高可行性 ${ }^{[19]}$ 。电磁发电技术是一种有效的能量收集 方法, 具有输出功率高、能量密度大、电源性能好 等特点 ${ }^{[20]}$ 。系统采用了一种电磁式振动能源采集结 
构对振动能量进行收集, 能量采集模块结构如图 3 所示。

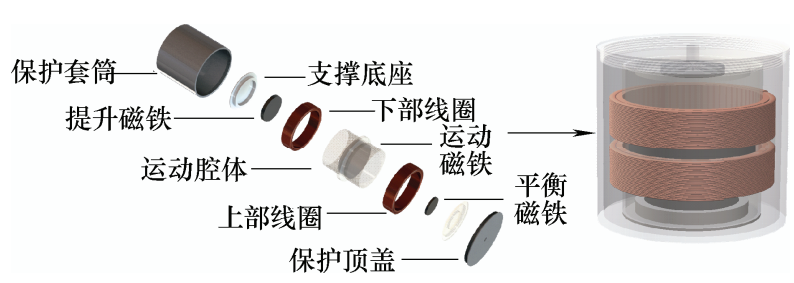

图 3 能量采集模块结构图

发电结构由磁悬浮系统、感应线圈、支撑与保 护结构等多个部分组成。相较于传统的弹簧-质量阻尼系统, 由磁力约束的阻尼系统具有更高的灵敏 度, 能够实现振动能量高效收集。使用 ANSYS Maxwell 软件对能量采集模块运行过程中磁场进行 仿真。如图 $4 \mathrm{a}$ 所示, 在静止状态下, 运动磁体保 持在中间平衡位置。感应线圈磁通量不变。当受到 外界振动激励后, 磁铁围绕平衡位置振荡。如图 $4 \mathrm{~b}$, $4 \mathrm{c}$ 所示, 在磁铁上下运动的过程中, 感应线圈磁 通量发生变化, 根据法拉第电磁感应定律, 产生感 应电动势与感应电流。

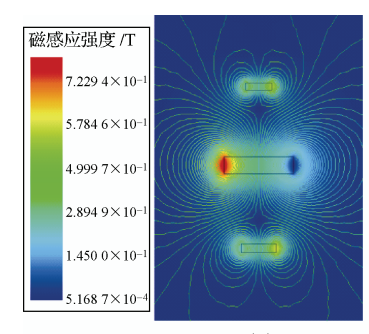

(a)

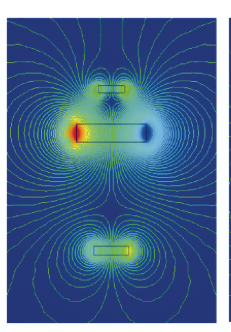

(b)

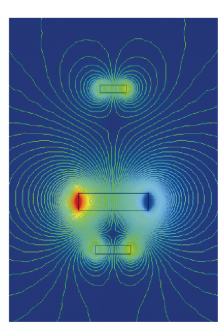

(c)
图 4 能量采集模块运行时内部磁场变化

\section{2 能量管理电路设计}

煤机设备工作环境恶劣, 系统受到的外界振动 具有很强的不确定性。在此种振动激励条件下, 能 量采集模块产生的电能大小有很大差异, 无法保证 稳定持续输出。若直接给节点电路供电, 除无法正 常驱动电路外还会给电路造成一定程度的损害。若 将产生的电能直接存入电池中, 在充电过程中会产 生很大的能量损失且会影响电池寿命 ${ }^{[21]}$ 。

为了解决上述问题, 设计能量管理电路对电 能进行有效收集。能量采集模块产生的电能经整 流、稳压后, 在储能电容中进行寄存, 当电容电 压值达到阈值上限后, 释放存储的电能对电池进 行充电, 达到阈值下限后停止电荷释放并进行再 次蓄积, 准备下次释放。相比微弱电流直接给电 池充电的方式, 采用积攒释放、平稳电流的充电
方法能够有效提高能源采集效率。图 5 为能量管 理电路工作流程图。

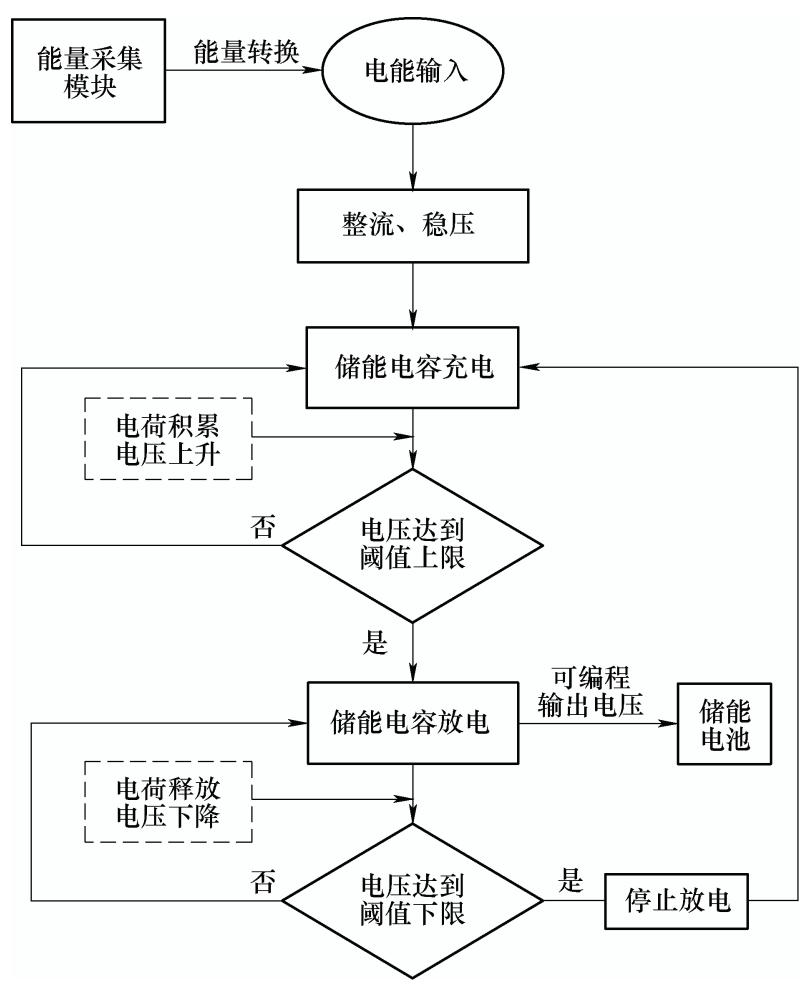

图 5 能量管理电路工作流程图

\section{3 主控电路设计}

主控电路是采集节点的核心, 用以控制各个模 块运行并进行数据处理与智能判断, 选用 TI 公司生 产的超低功耗单片机 MSP4305438A 作为系统主控 芯片, 该芯片功能丰富, 处理能力强、运算速度快, 满足系统数据处理需求; 在 $-40 \sim+80{ }^{\circ} \mathrm{C}$ 温度范围 内都可正常工作, 达到监测系统对工作环境温度要 求; 运行模式下工作电流为 $230 \mu \mathrm{A} / \mathrm{MHz}$, 满足系 统低功耗需求。图 6 为主控电路原理图。

\section{4 传感器模块设计}

传感器模块用以获取煤机设备状态信息, 为进 一步降低系统功耗, 选取数字型传感器而非模拟型 传感器进行数据采集。系统选用超低功耗 ADXL345 数字型三轴加速度传感器对振动信号进行测量。该 传感器能够精确测量重力加速度, 分辨率可以达到 $3.9 \mathrm{mg} / \mathrm{LSB}$, 正常工作时功耗为 $0.429 \mathrm{~mW}$, 待机时 功耗低至 $0.25 \mu \mathrm{W}$, 满足系统振动监测需求。选用 数字式温度传感器 DS18B20 进行设备温度监测, 该 传感器具有功耗低、精度高、抗干扰能力强等优点。 测量精度可达到 $0.5{ }^{\circ} \mathrm{C}$, 满足系统温度监测需求。

图 7 为传感器模块电路原理图。

\section{5 无线传输模块设计}

ZigBee 无线通信技术以功耗低、时延短、网络 容量大等优点, 广泛应用于工业自动化、消防安全、 


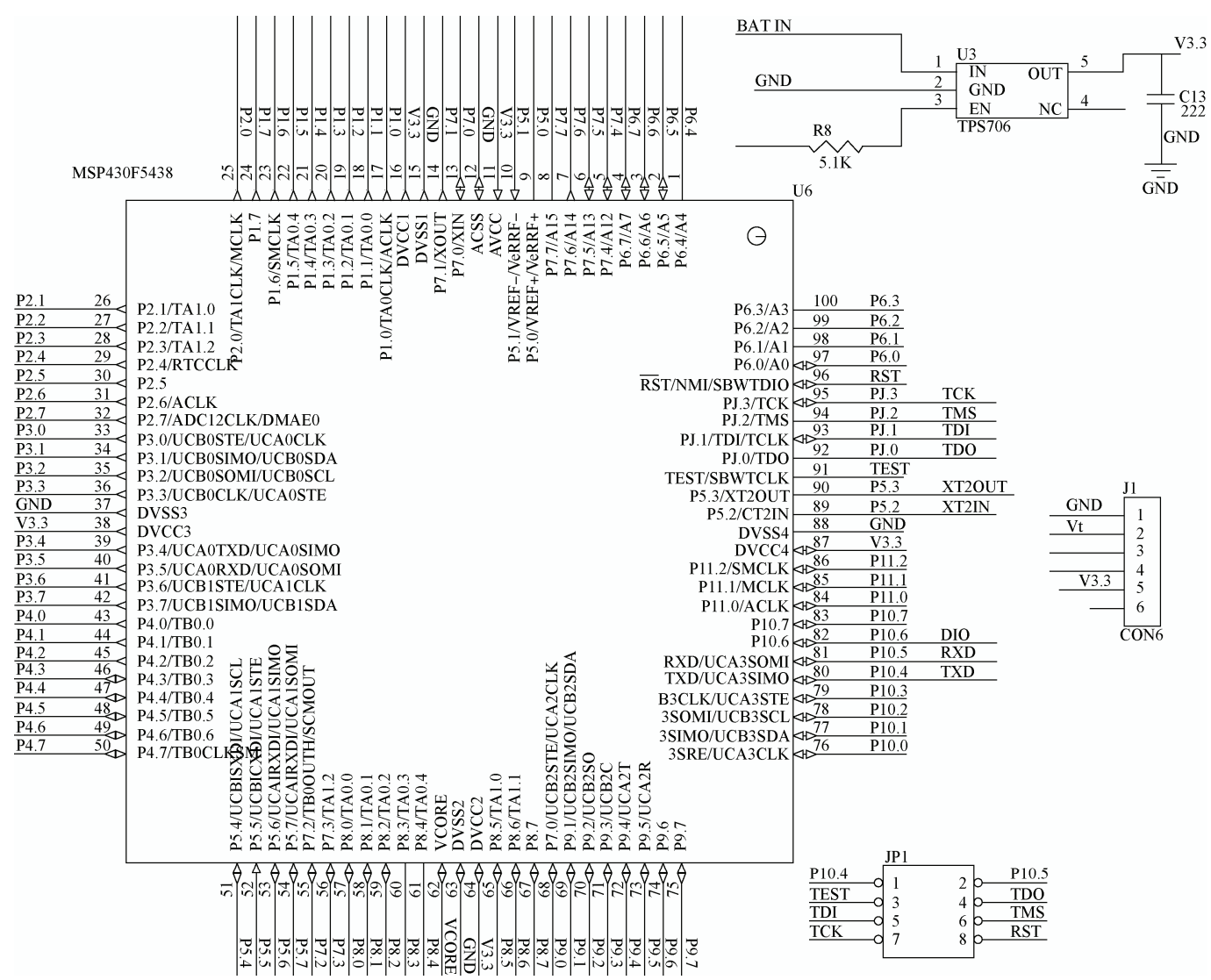

图 6 主控电路原理图

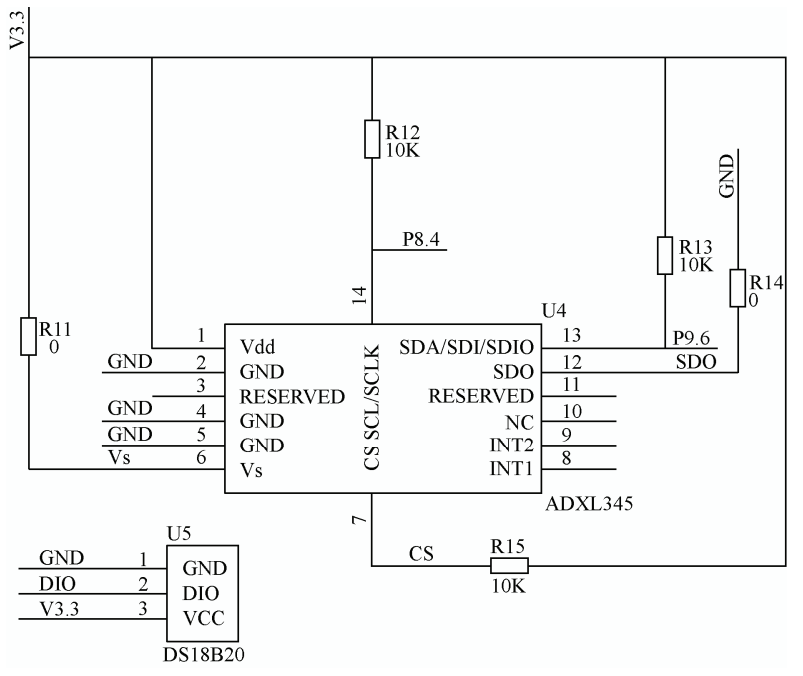

图 7 传感器模块原理图

环境保护、遥感勘测、煤矿、石化等领域 ${ }^{[22]}$ 。系统 中选用 CC2530 芯片作为无线通信模块主芯片。该 芯片可设置多种运行模式, 运行模式之间的转换时 间极短, 满足系统数据无线传输要求。图 8 为无线 传输模块原理图。

\section{6 电路集成设计}

考虑到系统结构尺寸、无线收发与一体集成的 需求, 将系统 PCB 分为主板和天线电路板两部分展 开设计。主板主要包括能量采集电路、主控电路、 传感器电路和程序下载口, 根据外壳形状设计为圆
形便于与其他模块的集成; 天线板主要包括射频芯 片和 PCB 天线, 置于主板上方, 便于传输数据。加 工完成的电路如图 9 所示。

\section{3 系统软件设计}

监测系统采用主控芯片管理系统各模块之间的 工作与通信, 使用上位机进行监测数据的实时显示、 存储与预警。故在软件层面需要完成主控芯片程序 算法与系统上位机软件的设计。

\section{1 主控程序设计}

当煤机设备关停时，系统获取到的数据基本 是无监测意义的“零状态值”, 传输此类数据, 不 仅浪费收集的振动能量并且会增大数据的午余 率。考虑到此种情况, 除传感器信号处理程序、 无线收发模块控制程序之外，在处理程序中加入 智能判断算法以提高监测效率，降低系统功耗。 如图 10 所示, 主控芯片对传感器模块采集到的传 感信号进行处理并进行数据分析与比对, 若实时 传感参数超过系统设定阈值，无线发送模块被唤 醒并向数据接收器发送数据, 之后根据实时监测 值判断系统下一步是否继续进行数据发送。通过 智能判断, 排除无用数据发送, 从而降低系统功 耗, 减少数据冗余。 


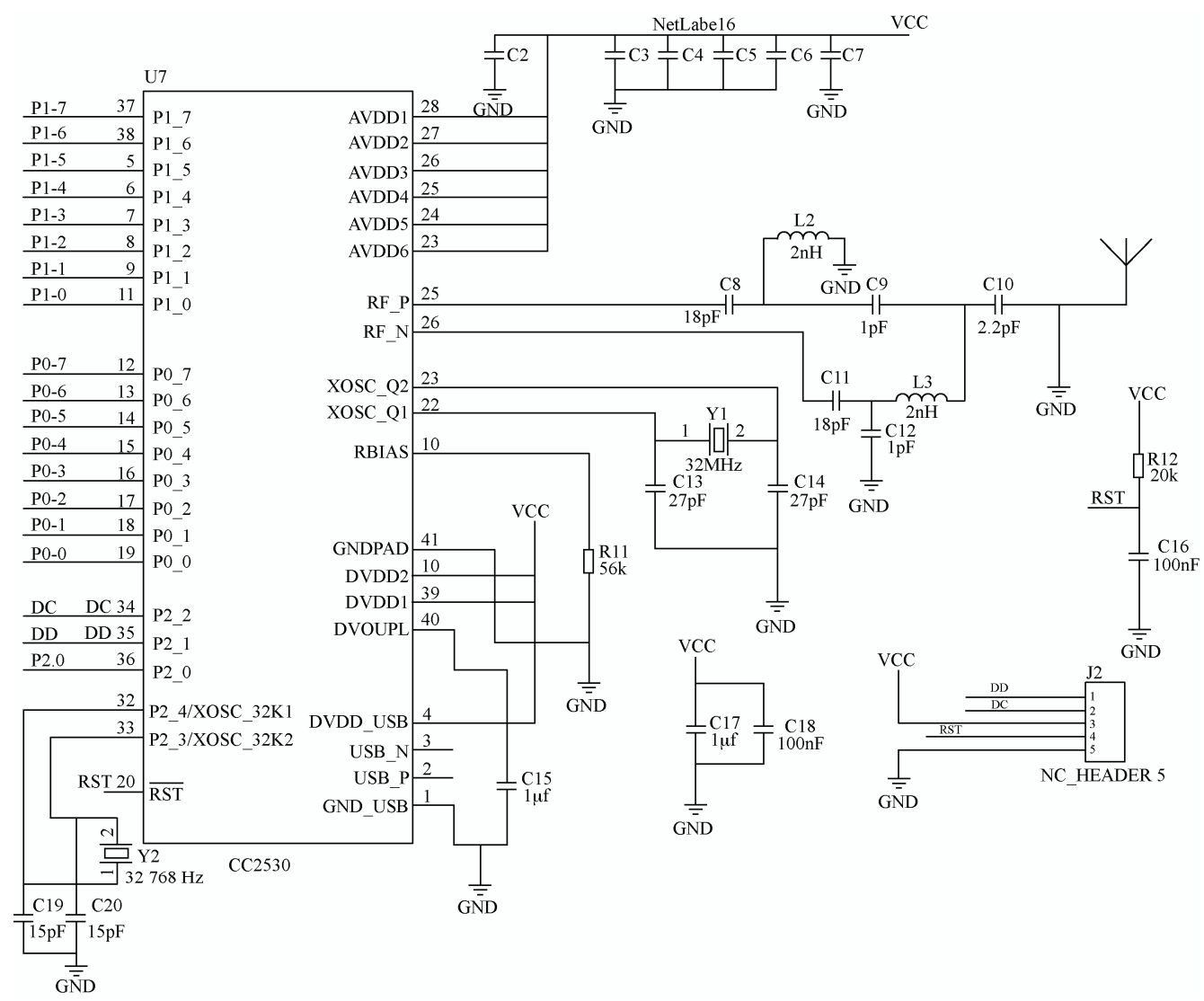

图 8 无线传输模块原理图

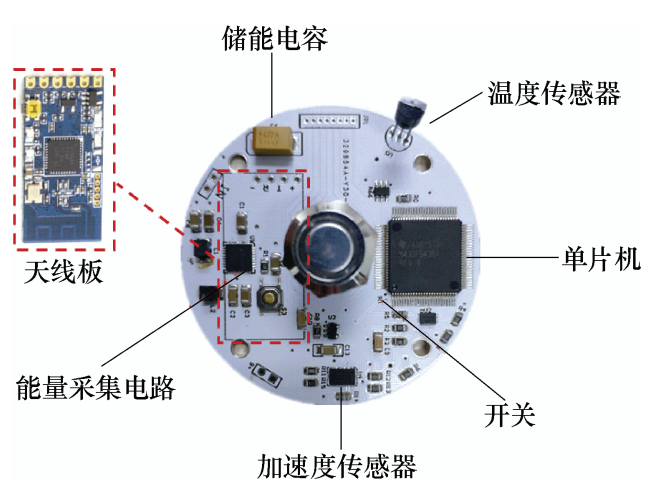

图 9 采集节点电路

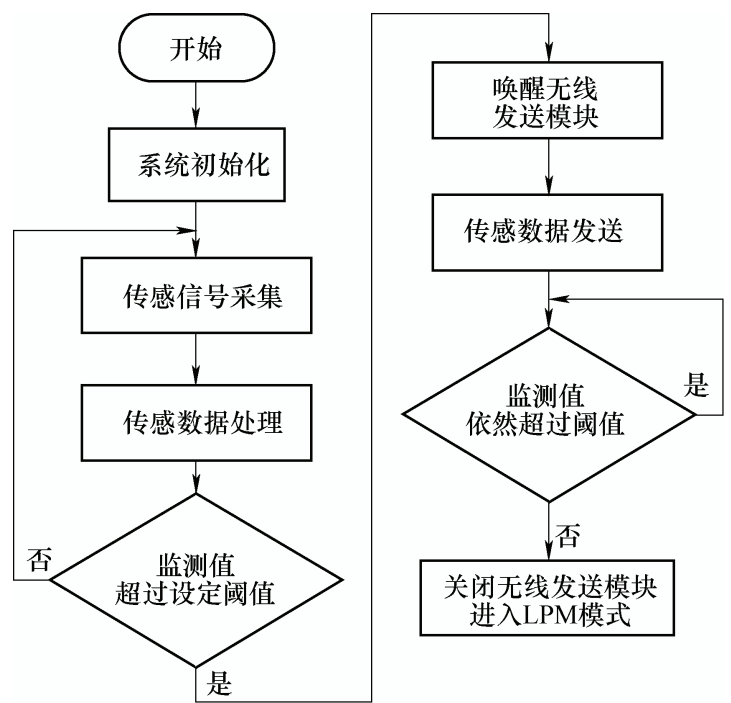

图 10 传感数据处理流程图

\section{2 无线传输模块程序设计}

无线传输模块包括装载于各个采集节点内的无 线发送模块与数据接收器中的无线接收模块, 系统 通过 Z-Stack 协议栈建立ZigBee 网络。如图 11 所示, 单片机将传感器数据处理完成之后, 发送指令给无 线发送模块, 将其从低功耗模式唤醒并开始进行数 据传输, 传感数据经获取、打包、发送、接收、融 合、转化后传入数据接收器, 最终采用 USB 通信方 式将数据传入上位机中，完成监测数据传输。

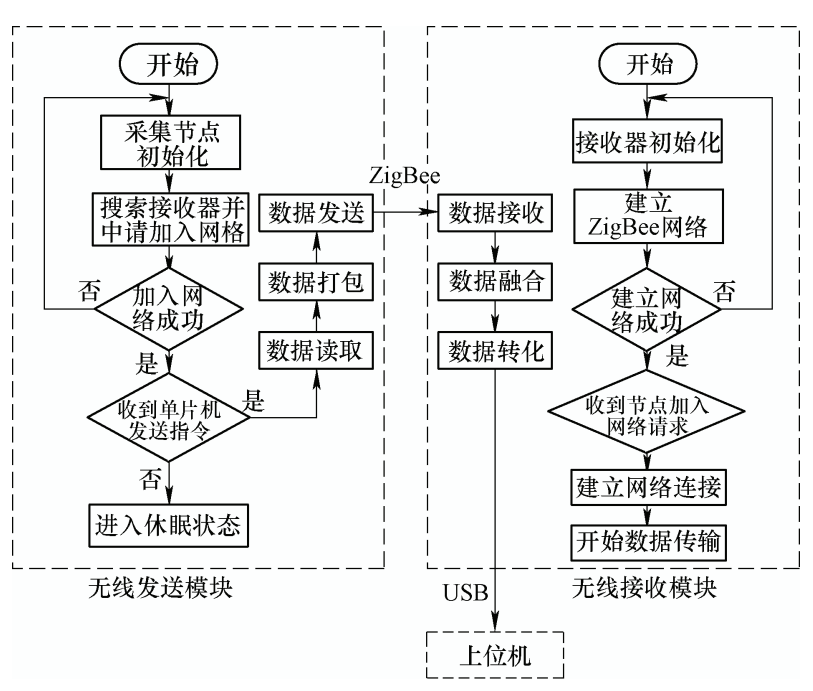

图 11 无线传输模块工作流程图 


\section{3 系统上位机设计}

系统通过上位机软件实现传感信号的实时监 测和预警, 完成系统人机交互。如图 12 所示, 数 据接收器将获取的采集节点数据传入上位机, 经数 据解码、节点号识别、数据库更新, 对信号进行处 理、分析、存储。最终将处理后的设备状态信息在 控制面板进行实时显示并在监测值超出國值后进 行报警。控制面板可直接调整报警阈值大小，以满 足各类煤机设备在不同工作环境下监测需求。通过 数据库可实现监测数据的存储、查看和调用, 为研 究煤机设备运行规律、解析故障发生原因提供数据 支持。

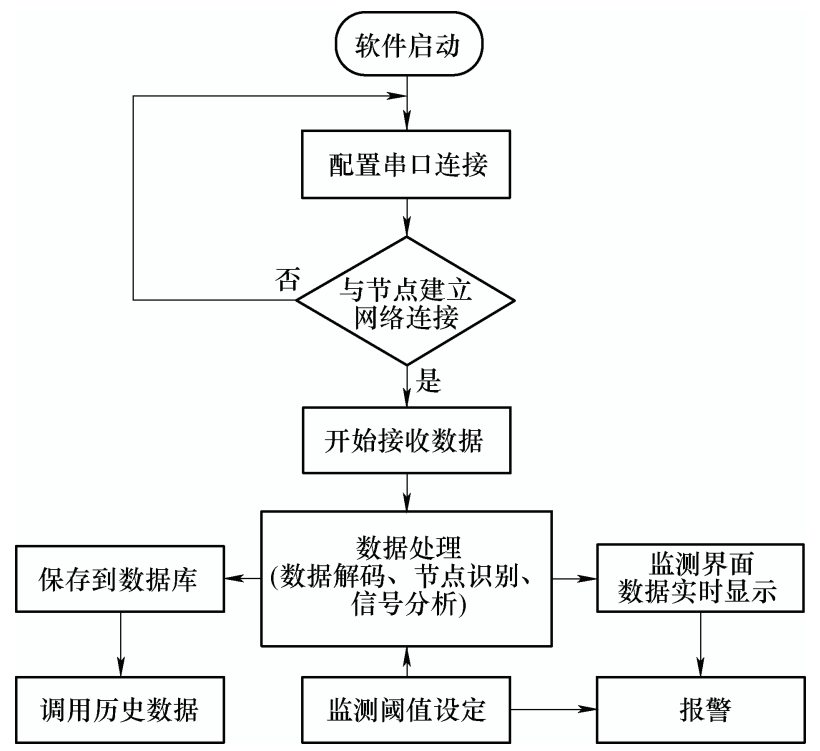

图 12 系统上位机工作流程图

4 系统集成与测试

\section{1 一体化集成}

如图 13a 所示，将能量采集模块、电路模块、 无线传输模块、开关等按设计路线封装进壳体中, 最后灌封保护胶, 实现对内部各个模块的保护。最 终完成的采集节点样机如图 13b 所示。

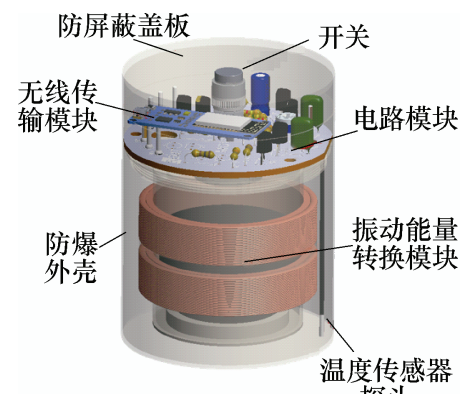

(a) 集成示意图

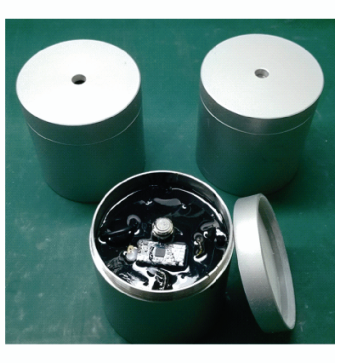

(b) 采集节点
图 13 采集节点一体化集成

\section{2 能耗测试与分析}

搭建如图 14 所示的测试平台, 对能量采集模块 输出特性进行测试。采用信号发生器与功率放大器 控制振动台模拟不同振动环境，使用数字源表 Keithley 2611B 与示波器测试能量采集模块输出信 号。图 15 为输出电压与输出功率随负载电阻的变 化, 可以看出, 当负载电阻为 $7.7 \mathrm{k} \Omega$ 时, 与能量采 集模块实现最佳阻抗匹配, 达到最大输出功率。

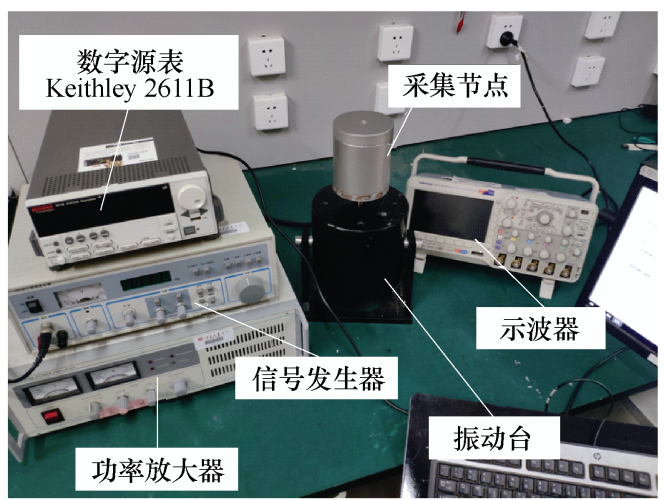

图 14 测试平台

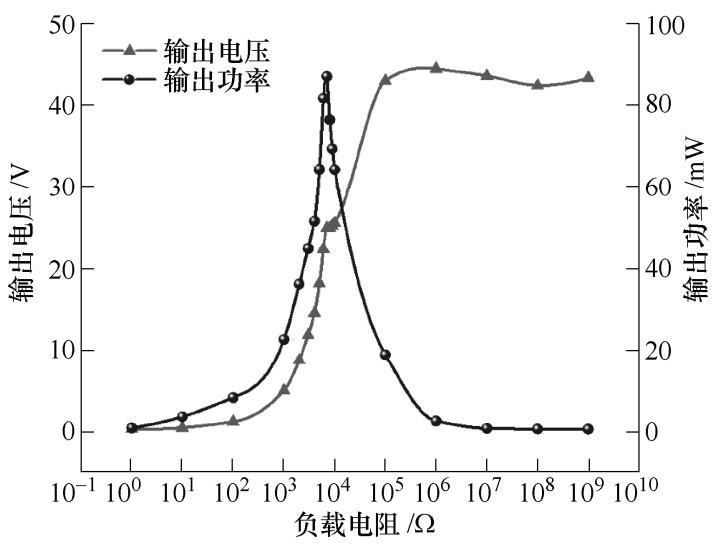

图 15 输出特性与负载电阻关系

确定最佳匹配电阻后，研究不同频率、振幅对 输出特性的影响。从图 16 可以看出, 该模块适用于 低频振动环境, 输出功率在谐振频率 $12.5 \mathrm{~Hz}$ 下达 到最大值。而且随着振动幅度逐渐增大, 输出功率 随之增大。

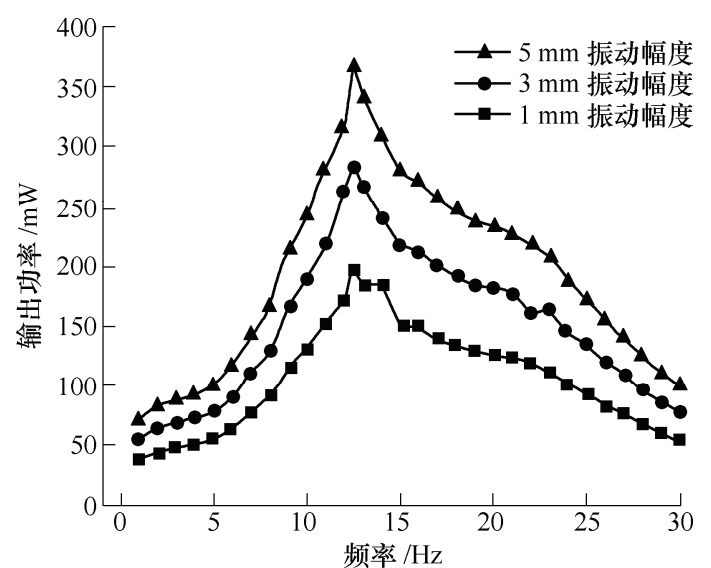

图 16 不同振幅、频率振动激励下输出功率 
保持负载电阻与振动频率不变, 测试能量采集 模块输出电流随外界振动幅度变化情况如图 17 所 示, 输出电流大小随振幅增加而增大。

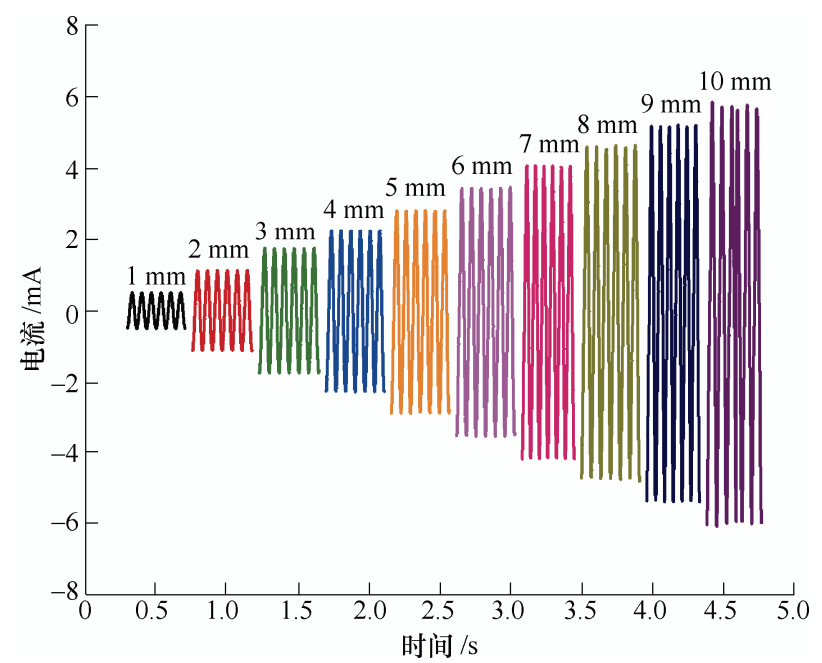

图 17 不同振幅下输出电流

对能量采集模块在不同的冲击加速度下输出功 率进行测试。如图 18 所示, 当加速度在 $0 \sim 2 g$ 范 围内, 输出功率约为 $14.4 \mathrm{~mW}$, 随着加速度增加, 能量采集模块产生的电能逐渐增大, 当加速度达到 $13 \mathrm{~g}$, 输出功率达到 $378 \mathrm{~mW}$ 并趋于稳定。

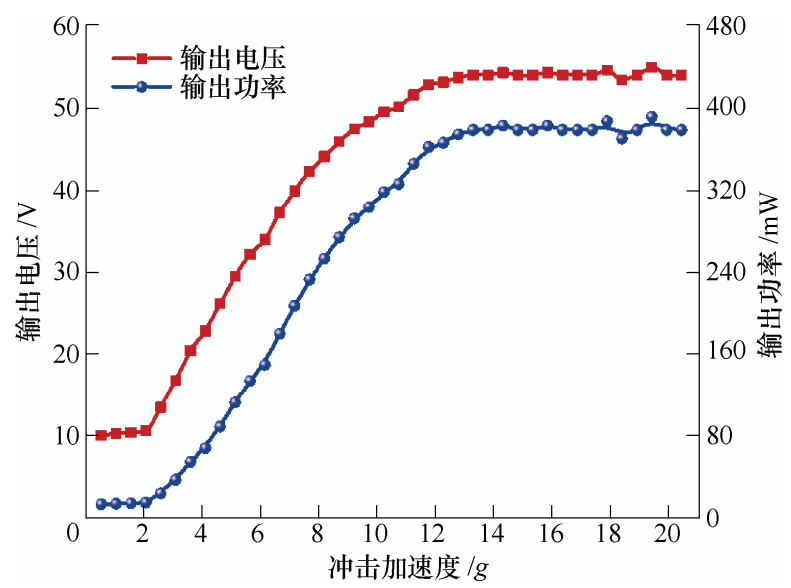

图 18 输出特性与冲击加速度关系

设置能量管理电路阈值下限为 $5 \mathrm{~V}$ 以满足锂电 池充电需求。经多次测试充电并比对充电效果, 最 终设置阈值上限为 $6 \mathrm{~V}$ 。如图 19 所示, 在外界振动 激励下, 能量采集模块产生电能输入能量管理电路, 电路中的储能电容电压开始上升, 当电压达到 $6 \mathrm{~V}$ 后, 释放电能为电池充电; 当电压下降到 $5 \mathrm{~V}$ 后, 停止电能释放, 并再次蓄积电荷等待下次释放。可 以看出, 能量采集电路能够实现电能的有效收集。

在系统各个功能模块中, 需要供电的单元主要 包括采集节点中单片机、传感器以及无线发送模块 (无线接收器通过 USB 由 PC 端供电)。如表 1 所示, 各模块在工作状态下总功耗为 $106 \mathrm{~mW}$, 而在天线

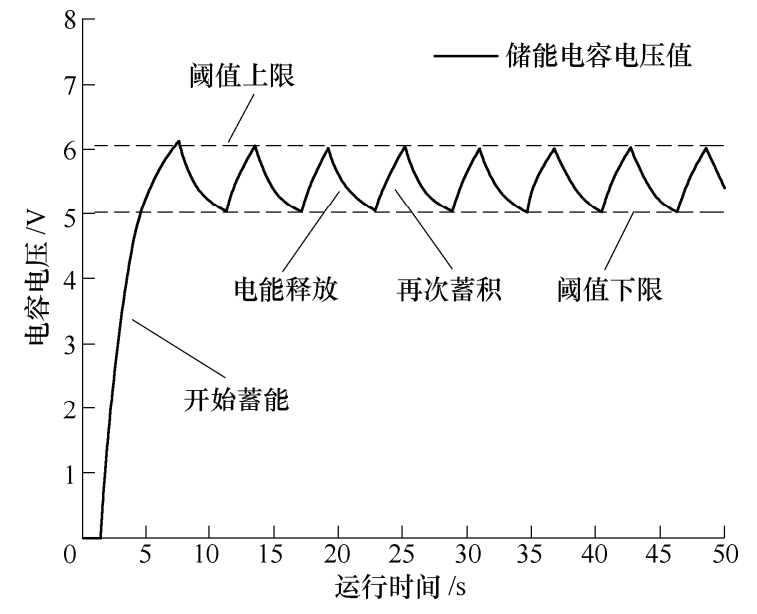

图 19 能量采集电路工作时储能电容电压值变化

休眠模式下, 功耗则低至 $12 \mathrm{~mW}$ 。能量采集模块输 出功率满足系统供电需求。

表 1 系统各模块功耗

\begin{tabular}{cccc}
\hline 功能模块 & 工作电压 $/ \mathrm{V}$ & 工作电流 $/ \mathrm{mA}$ & 功耗 $/ \mathrm{mW}$ \\
\hline 单片机 & 3.3 & 1.8 & 5.94 \\
温度传感器 & 3.3 & 1.5 & 4.95 \\
加速度传感器 & 3.3 & 0.13 & 0.429 \\
天线 & 3.3 & 28.7 & 94.71 \\
总计 & - & - & 106.029 \\
\hline
\end{tabular}

\section{3 实地试验}

在阳煤集团二矿进行系统现场试验, 如图 20 所示, 信号采集节点安装于大型皮带机驱动电机(图 20a)不同位置处(图 20b、20c、20d)以采集设备振动 能量与传感信号; 无线接收器与计算机端置于监控 室(图 20e)接收采集节点发出的无线信号, 信号采集 节点所在位置与接收器相隔距离 $32 \mathrm{~m}$ (图 20f)。系统 最终在上位机监测界面中获取到各个传感节点的监 测值(图 20g), 成功实现对煤机设备运行状态的实时 监测。

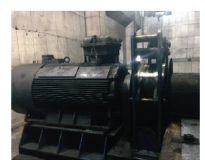

(a)

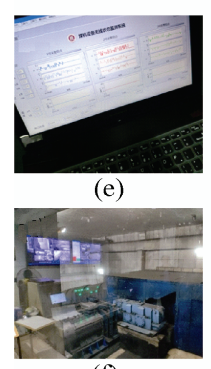

(f)

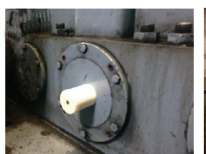

(b)

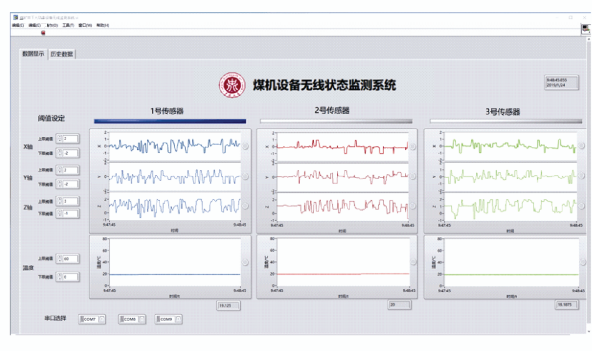

(g)
图 20 试验现场与上位机监测结果 


\section{5 结论}

设计并完成了煤机设备无线自供电状态监测系 统, 该系统在对煤机设备关键状态信息进行监测的 同时可收集设备振动能量为节点电路供电。本文着 重从自供电模块设计、系统硬件电路设计、系统软 件设计、系统一体化集成与测试几个方面介绍了监 测系统的设计思路与方法。测试结果表明, 系统能 够有效收集环境振动能量实现能源自供给, 能够精 准采集煤机设备振动、温度信号, 并通过 ZigBee 技术进行数据无线传输, 最终在系统上位机实现煤 机设备状态信息的实时监测与预警。

\section{参 考 文 献}

[1] HUANG Jianfeng, CHEN Guohua, SHU Lei, et al. WSNs-based mechanical equipment state monitoring and fault diagnosis in China[J]. International Journal of Distributed Sensor Networks, 2015(4): 1-14.

[2] 孙继平. 煤矿信息化与自动化发展趋势 [J]. 工矿自动 化, 2015，41(4): 1-5.

SUN Jiping. Development trend of coal mine informatization and automation $[\mathrm{J}]$. Industry and Mine Automation, 2015, 41(4): 1-5.

[3] 普亚松, 郭德伟, 张文斌. 故障诊断技术在煤矿机械设 备中的应用[J]. 工矿自动化, 2015，41(4): 36-39. PU Yasong, GUO Dewei, ZHANG Wenbin. Application of fault diagnosis technologies in coal mine machinery[J]. Industry and Mine Automation, 2015, 41(4): 36-39.

[4] CHEN Yanhua, OU Jin, MA Xuan. Research on coal mine safety wireless monitoring system based on bluetooth technology[J]. Advanced Materials Research, 2013, 765-767: 2061-2064.

[5] 王强, 张培林, 王怀光, 等. 基于优化分类的机械振动 信号压缩感知 $[\mathrm{J}]$. 振动与冲击, 2018, 37(14): 86-93. WANG Qiang, ZHANG Peilin, WANG Huaiguang, et al. Compressed sensing algorithm for machinery vibration signals based on optimal classification [J]. Journal of Vibration and Shock, 2018，37(14): 86-93.

[6] 王国彪, 何正嘉, 陈雪峰, 等. 机械故障诊断基础研究 “何去何从”[J]. 机械工程学报, 2013，49(1)：63-72. WANG Guobiao, HE Zhengjia, CHEN Xuefeng, et al. Basic research on machinery fault diagnosis - What is the prescription[J]. Journal of Mechanical Engineering , 2013, 49(1): 63-72.

[7] 曾超, 汤宝平, 肖金金, 等. 低功耗机械振动无线传感器 网络节点结构设计 $[\mathrm{J}]$. 振动与冲击, 2017, 36(14): 33-37.
ZENG Chao, TANG Baoping, XIAO Xin, et al. Low power node architecture design for mechanical vibration wireless sensor networks[J]. Journal of Vibration and Shock, 2017, 36(14): 33-37.

[8] CHENG Jie, GAO Dianwu , WANG Junfei , et al. Coal mine safety monitoring system based on ZigBee and GPRS[J]. Applied Mechanics and Materials，2013，422: 215-220.

[9] 张文栋, 薛晨阳. 煤矿机械工作状态监测系统研究 [J]. 振动测试与诊断，2018，38(5): 883-889.

ZHANG Wendong, XUE Chenyang. Research on working state monitoring system of coal mine machinery[J]. Journal of Vibration, Measurement \& Diagnosis, 2018, 38(5): $883-889$

[10] 张弛, 付贤鹏, 王中林. 摩擦纳米发电机在自驱动微系 统研究中的现状与展望 [J]. 机械工程学报, 2019, 55(7): 89-101.

ZHANG Chi, FU Xianpeng, WANG Zhonglin. Review and prospect of triboelectric nanogenerators in self-powered microsystems[J]. Journal of Mechanical Engineering, 2019, 55(7): 89-101.

[11] WANG Zhonglin. On Maxwell's displacement current for energy and sensors: The origin of nanogenerators[J]. Materials Today, 2017, 20(2): 74-82.

[12] KHALIGH A, ZENG P, ZHENG C. Kinetic energy harvesting using piezoelectric and electromagnetic technologies - state of the art[J]. IEEE Transactions on Industrial Electronics, 2010， 57(3): 850-860.

[13] XUE Chenyang, LI Junyang, ZHANG Qiang, et al. A novel arch-shape nanogenerator based on piezoelectric and triboelectric mechanism for mechanical energy harvesting $[\mathrm{J}]$. Nanomaterials, 2014， 5(1): 36-46.

[14] WANG Jie, LI Shengming, YI Fang, et al. Sustainably powering wearable electronics solely by biomechanical energy[J]. Nature Communications， 2016， 7: 12744.

[15] ZHANG Zengxing, HE Jian, WEN Tao, et al. Magnetically levitated-triboelectric nanogenerator as a self-powered vibration monitoring sensor[J]. Nano Energy, 2017, 33: 88-97.

[16] 黄庆卿, 汤宝平, 邓蕾, 等. 机械振动无线传感网络数 据分块无损压缩方法 [J]. 仪器仪表学报, 2015，36(7): 1605-1610.

HUANG Qingqing, TANG Baoping, DENG Lei, et al. Data block-based lossless compression for machine vibration wireless sensor networks [J]. Chinese Journal of Scientific Instrument，2015，36(7): 1605-1610.

[17] CHEN Xuexian, GUO Hang, WU Hanxiang, et al. Hybrid generator based on freestanding magnet as all-direction in-plane energy harvester and vibration sensor[J]. Nano 
Energy, 2018, 49: 51-58.

[18] ASKARI H, SAADATNIA Z, ASADI E, et al. A flexible hybridized electromagnetic-triboelectric multi-purpose self-powered sensor[J]. Nano Energy, 2018, 45: 319-329.

[19] SUN Shi, DAI Xuhan, WANG Kai, et al. Nonlinear electromagnetic vibration energy harvester with high efficient closed magnetic circuit[J]. IEEE Magnetics Letters, 2018, 9: 1-5.

[20] WANG Xin, WEN Zhen, GUO Hengyu, et al. A Fully packaged blue energy harvester by hybridizing a rolling triboelectric nanogenerator and an electromagnetic generator[J]. ACS Nano, 2016，10: 11369-11376.

[21] 刘大同, 周建宝, 郭力萌, 等. 锂离子电池健康评估和 寿命预测综述 [J], 仪器仪表学报, 2015, 36(1): 1-16.
LIU Datong, ZHOU Jianbao, GUO Limeng, et al. Survey on lithium-lion battery health assessment and cycle life estimation [J]. Chinese Journal of Scientific Instrument, 2015, 36(1): 1-16.

[22] YANG Wei, ZHANG Yu, LIU Yang. Constructing of wireless emergency communication system for underground coal mine based on WMN technology[J]. Journal of Coal Science and Engineering, 2010， 16(4): 441-448.

作者简介: 高扬, 男, 1995 年出生。主要研究方向为微能源技术与无线 传感器网络。

E-mail: 495250229@qq.com

丑修建(通信作者), 男, 1979 年出生, 博士, 教授, 博士研究生导师。 主要研究方向为智能传感、先进制造、电子测试等领域。

E-mail: chouxiujian@nuc.edu.cn 